b) sur la pente du gradin, il se dépose de temps en temps et çà et là des matériaux irréguliers, même dans les régions à roche homogène, de telle sorte qu'il se forme de nouveaux gradins d'accumulation.

c) les différentes périodes d'érosion glaciaire ont provoqué des irrégularités dans le lit des rivières, lesquelles n'ont pas encore été effacées par l'érosion fluviatile actuelle. Il faut tenir compte surtout dans le profil longitudinal des surcreusements glaciaires qui nous sont cachés par l'accumulation fluviatile et qui n'ont été découverts que récemment par les recherches sismiques modernes.

d) des phénomènes tectoniques, même de faible envergure, provoquent au bord et à l'intérieur des Alpes de nouveaux gradins et transforment les conditions d'érosion et d'accumulation.

La nivellation de ces gradins très différenciés et se reformant constamment ne se fait pas d’après une loi de nivellation générale, mais elle est elle-même très variée. Ainsi, la théorie de la migration des gradins - leur déplacement rétrograde sans changement de forme le long de vallées entières - est trop schématique.

Cette étude invite à ne pas s'intéresser uniquement aux migrations des gradins et aux phases de soulèvement qui leur sont tout simplement liées, mais aussi à se tourner vers la grande richesse de formes et de phénomènes en sa totalité qu’offrent les vallées des Alpes.

\title{
EINE ALTE KARTE VON NEU ENGLAND
}

\section{Hans BoEsch}

Dank dem Entgegenkommen der Firma Kümmerly \& Frey, Bern, konnte aus dem «Theatrum Orbis Terrarum sive Atlas Novus», in erster Auflage 1635 in den Offizinen des WILLEM Janszoon BlaEU in Amsterdam erschienen, ein Teil des Kartenblattes «Nova Belgica et Anglia Nova» hier reproduziert werden. Die Originalkarte stammt aus der Auflage 1660 und wurde von Dr. D. BrunnschweIler, Zürich, freundlicherweise zur Verfügung gestellt. Die Reproduktion konnte aus dem 1956 erschienen Werke des Verfassers «USA — Die Erschließung eines Kontinentes» (bei Kümmerly \& Frey, Bern) übernommen werden.

Um 1600 verschob sich das Zentrum der kartographischen Produktion von Antwerpen (Abraham Ortel oder Ortelius, Gerard Kraemer oder Mercator) nach Amsterdam. Die Holländische Ost- und Westindische Kompanie als Auftraggeber für genaue Seekarten und die im Kolonialhandel reich gewordenen Kaufleute als Käufer von kostbaren Atlanten und vollendet gestalteten Wandkarten waren hier die Voraussetzung für eine blühende Entwicklung einer vielseitig orientierten Kartographie und Kartendruckerei.

R. A. Skelton (in: Decorative Printed Maps of the 15th to 18th Centuries, London 1952, p. 58 ff.) berichtet uns über den Autor der vorliegenden Karte Folgendes: «Ungefähr 1596 begann Willem Janszoon BlaEU sich als Hersteller von Instrumenten und Globen, etwas später als Kupferstecher und Drucker zu betätigen.... Seine frühesten Publikationen waren Tabellen der Deklination, Plattkarten und Segelanweisungen für die Lotsen; seine Verdienste um die Seefahrt wurden durch finanzielle Unterstützung von Seiten der Generalstaaten und durch seine Ernennung (1633) zum Kartographen der Republik anerkannt. Nicht geringere technische Begabung zeigte BlaEU bei der Einrichtung seiner Druckerei. In den Annalen der Typographie ist BlaEU bekannt als der Erfinder einer Druckerpresse, welche die Produktion um einen Viertel zu vermehren gestattete. Eine bekannte Beschreibung seiner Druckerei, welche er in den Jahren 1616-1637 - ein Jahr vor seinem Tode - errichtet hatte, hebt die auf das höchste entwickelte Betriebsorganisation des Unternehmens von BLAEU und seinen Söhnen hervor. FILIP vON ZESEN, welcher Amsterdam anno 1663 besuchte, schrieb darüber: < An der Blumengracht . . . findet man die weitbekannte Druckerei von Johan Blaeu. Sie ist mit neun Typenpressen, welche nach den neun Musen benannt sind, sechs Pressen für Kupferplatten-Druck sowie einer Typengießerei ausgerüstet> , 》

Neben der Firma von BLAEU und seinen Söhnen war um diese Zeit in Amsterdam die Kartendruckerei des flämischen Kupferstechers Jodocus Hondius bekannt, welche später von seinem Schwiegersohn JAN JANSSON übernommen wurde. In beiden Fällen handelt es sich, wie aus der obigen Beschreibung hervorgeht, um bedeu- 
tende kaufmännische Unternehmen. Die publizierten Karten beruhten im wesentlichen auf mehr oder weniger sorgfältigen Kompilationen aller zugänglichen Unterlagen von oft recht verschiedener Genauigkeit, wobei sich die verschiedenen Kartenfirmen gelegentlich gegenseitig unterstützten. Häufiger waren jedoch die Fälle, wo eine Firma die Karten der anderen einfach kopierte. «Im Jahre 1608, dem Erscheinungsjahr der großen Weltkarte von Hondius, in welcher er zahlreiche Angaben aus der BlaEU'schen Weltkarte von 1605 mit verwendete, appellierte BLAEU an die Generalstaaten und ersuchte um Schutz vor Personen, die ihn durch das Kopieren von Karten materiell schädigten》(SKELTON).

BlaEU publizierte, wie schon gesagt, seinen «Novus Atlas» im Jahre 1635; Jansson folgte 1638 mit seinem «Nieuwen Atlas ofte werelt Beschrijvinge». Von diesen beiden Atlanten erschienen in den folgenden Dezennien zahlreiche Neuauflagen, die immer umfangreicher wurden. Janssons Atlas schwoll bis 1658-61 auf elf Bände an, und die 1662 unter dem Titel «Atlas Maior»gedruckte Neuauflage des BLAEU'schen Werkes zählte in der französischen Ausgabe sogar zwölf Bände. Auf die Qualität der Karten, vor allem auf die sorgfältige Nachführung in der Zwischenzeit bekannt gewordener Neuentdeckungen, wurde bei diesen rein kommerziellen Zielen folgenden Unternehmungen geringer Wert gelegt - ganz im Gegensatz etwa zu den früheren Karten eines Mercator. Die hier reproduzierte Karte von 1660 unterscheidet sich, wie eine sorgfältige Prüfung ergab, nur in der Änderung des Erscheinungsjahres von der bei SKelton reproduzierten Erstausgabe von 1635.

Besonders bemerkenswert ist in diesem Zusammenhange der Titel des Kartenblattes «Nova Belgica et Anglia Nova» sowie die zweifache Bezeichnung des Gebietes der holländischen Kolonien mit «Nieu Nederlandt» und «Novum Belgium». (Auf unserer Reproduktion fehlt der Titel, und die genannte Gebietsbezeichnung ist nur teilweise wiedergegeben.) 1610 wurde die Kolonie Neu-Niederlande gegründet, welche seit 1621 der Holländisch Westindischen Kompanie eingegliedert war. Unser Kartenausschnitt zeigt unter anderem Nieu Amsterdam (das heutige New York) und Fort Orange (Albany). Diese Kolonie verloren die Holländer 1664 an die Engländer. Da erst im westphälischen Frieden, 16+8, die Abtrennung der nördlichen Provinzen und damit die niederländische Souveränität international anerkannt wurde, ist die zusätzliche Bezeichnung «Novum Belgium» auf der Ausgabe von 1635 leicht verständlich. Hingegen zeugt es von einer unsorgfältigen Bearbeitung von Neuausgaben, daß noch in der Ausgabe von 1660 die Bezeichnung Nova Belgica stehen blieb!

Einen ähnlichen Eindruck gewinnen wir bei einer sorgfältigen Prüfung des englischen Kolonisationsgebietes, Nova Anglia oder Nieu Engeland. 1620 wurde die erste Niederlassung, New Plymouth, gegründet, die auf der Karte als Nieu Pleimouth auch eingezeichnet ist. Um 1640 bestanden zahlreiche Siedlungen schon entlang der ganzen Küste Neu Englands: 1630 Boston als Siedlungszentrum der Massachusetts-BayCompany, 1633 Connectictut, 1636 Rhode Island. 1643 wurden die Kolonien von Massachusetts, New Plymouth, Connectitut und Newhaven als «Vereinigte Kolonien von Neu England» zusammengefaßt. Von all dem findet man auf der Karte keine Spur.

Skelton weist auf Grund genauer Studien darauf hin, daß BlaEu für die Karte offenbar eine aus dem Jahre 1614 stammende Manuskriptkarte von A. BLock verwendet habe und später Nachträge der 1630-er Ausgabe der Karte «Nieuwe Wereldt» von J. DE LAET entnahm. DE LAET war Direktor der Holländisch Westindischen Kompanie und außerdem persönlich an den Siedlungsunternehmungen im Hudsontal interessiert. Seine Karte (1630) ist in J. WInsor « Narrative and Critical History of America», Vol. IV, London 1886, reproduziert; sie zeigt tatsächlich bis in Einzelheiten eine gute Übereinstimmung mit der Karte von BLAEU.

Überblickt man die Karte als Ganzes, so fällt in erster Linie auf, daß ihr linker - südlicher - Teil mit den Küstengebieten von Neu England und Neu Niederland 
in sich wohl proportioniert ist. Das Innere erscheint jedoch gegenüber der Küstenzone stark verschoben. Der «groote Rivier von Nieu Nederlandt» ist, wenn auch stark verzeichnet, der St. Lawrence River mit den Niagara Fällen («de groote afval») und der «Lacus Irocoisiensis» der heutige Lake Champlain, der in Wirklichkeit in der direkten, nördlichen Fortsetzung des Hudsontales liegt. BlaEU scheint die vorhandenen, guten französischen Kartenunterlagen für das kanadische Gebiet nicht verwendet zu haben. Dies ist erstaunlich, wenn wir an die ausgezeichneten Karten Kanadas von Champlain (1612 und vor allem 1632) denken. Freilich ist auch bei Champlain die gegenseitige Lage von Lake Champlain und Hudson unrichtig, die Verzerrung hält sich aber in gewissen Grenzen. Eine Durchsicht von Karten der ersten Hälfte des 17. Jahrhunderts zeigt ganz allgemein, daß diese Nahtlinie zwischen den französischen, englischen und niederländischen Kolonisationsräumen kartographisch nicht überbrückt werden konnte. Für die einzelnen Kolonisationsräume waren wohl verhältnismäßig gute Karten vorhanden, doch konnten diese nicht zur kartographischen Darstellung eines größeren Raumes zusammengefügt werden.

\title{
AN OLD MAP OF NEW ENGLAND
}

The original of the map, reproduced from the author's book "USA - Die Erschließung eines Kontinentes» (1956, Kümmerly \& Frey, Bern), comes from a late (1660) edition of W. J. BLAEU's world atlas "Theatrum Orbis Terrarum sive Atlas Novus", first published in 1635 in Amsterdam. The sheet, from which the reproduction was taken, bears the title "Nova Belgica et Anglia Nova". Since the Netherlands were not definitely recognized as a sovereign nation until 1648, this title is correct in the 1635 , but not in the 1660 edition. The cartographic content of the two editions is identical, and therefore the 1660 edition is outdated as far as settlements and the interior are concerned. The coast line, on the other hand, is fairly accurate. The areas adjacent to the coast were taken from maps made by de LAET (1630) and BLOCK (1614). The interior, after early French maps is very conjecturial. The two parts do not fit together, a common feature in most early $17^{\text {th }}$ century maps.

\section{ÜBER EINE ÜBERSCHIEBUNG DER TERTIÄREN SIWALIKS ÜBER DAS REZENTE GANGES ALLUVIUM IN OSTNEPAL}

\author{
Toni Hagen
}

Mit 3 Abbildungen

Seit langem sind junge und allerjüngste tektonische Bewegungen aus dem Himalaya bekannt, so Hebungen und Schiefstellungen pleistozäner See- und Flußablagerungen im Kaschmirtal (Karewa Serie) bis zu $1800 \mathrm{~m}$. Auch wurden schon verschiedentlich Überschiebungen von älteren Himalaya Formationen über pleistozäne Ablagerungen gemeldet. Der Geologe H.M. LAHIRI des «Geological Survey of India «berichtete sogar von einer Überschiebung der tertiären Siwalikformationen (Konglomerate der Obersiwaliks) über das horizontal gelagerte GangesAlluvium bei Ambala und Hoshiarpur im Punjab.

H. M. LAhIRI starb, und infolge mangelhafter Beschreibung konnte der betreffende Aufschluß später nicht mehr lokalisiert werden. Die Überschiebung wurde in der Folge sogar angezweifelt.

Dem Verfasser war es während der fünfmonatigen Expedition 1955/56 in Ostnepal vergönnt, eine eindeutige Überschiebung von hellgrauen Sandsteinen der Mittelsiwaliks (Tertiär) über den obersten, rezenten Schutt des Ganges Alluviums, zu finden. Der Aufschluß liegt bei Koordinate $87^{\circ} 30^{\prime} / 26^{\circ} 46^{\prime}$, am Oberlauf des Lohandra Khola, ca. $1500 \mathrm{~m}$ westnordwestlich des Dörfchens Kherwa. Er ist von Biratnagar aus während der Trockenzeit per Jeep in einem Tag gut erreichbar. Die Überschiebung ist an einem frisch angeschnittenen Terrassenrand von ca. $15 \mathrm{~m}$ Höhe besonders gut aufgeschlossen. 
